\title{
Hyperchaos in acetylcholinesterase enzyme systems
}

\begin{abstract}
Burst generation via a complex bifurcation scenario is discussed using a two compartments model of an enzyme system with substrate inhibition kinetics affected by the production of hydrogen ions accompanying the reaction (e.g. acetylcholinesterase enzyme system). Evidences are given to support the existence of homoclinicity associated with this complex dynamics, including the generalised criterion developed by Rossler et al. [1] for the application of Sil'nikov's theorem in the case of four-dimensional systems. Complex bistabilities are observed in certain regions, and the structure of some attracting sets occurring near homoclinic orbits are discussed. The results support the use of such fundamental models for different dynamical modes generation and analysis. The results relate to the transition of small and large frequency oscillations to periodic bursting and vice versa in excitable cells and many biophysical systems.
\end{abstract}

Keyword: Periodic burst generation; Bifurcation; Acetylcholinesterase enzyme; Burst generation 